\title{
Lantern Asparagus
}

Dr. Emily Ruth Rutter

2 Bunches

1 Pint

2 Tablespoons

2 Tablespoons

1/2 Cup

1 Teaspoon
Asparagus

Shiitake mushrooms

Sesame oil

Butter

Soy sauce

Black pepper

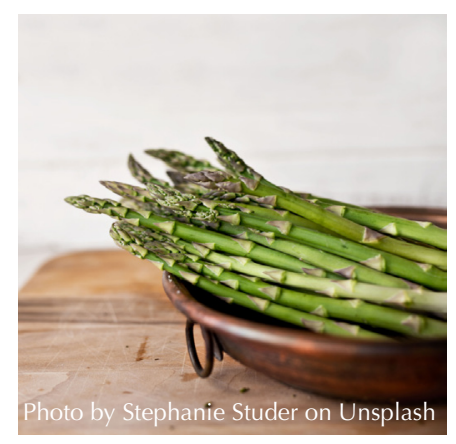

Description: Bundle of aspargus sits in bowl on right side, cut off by picture. The bowl is on a wooden cutting board.

This recipe is inspired by Lantern, a restaurant in Chapel Hill, N.C., where I worked for several years.

Typically, a soft-boiled egg would be placed on top, but I am squeamish about egg yolks (I know, I know).

1. Wash the asparagus and the shiitakes.

2. Cut off the stems of the asparagus and slice the spears diagonally into inch-long pieces.

3. Sauté the asparagus on medium heat along with the sesame oil for approximately 5 minutes.

4. Add the rinsed shiitake mushrooms, and sauté these vegetables together with the soy sauce and butter for approximately 10 minutes.

Serve as a side dish with friends or family who take as much pleasure savory vegetables as you do. 